Article

\title{
Beta-Glucans Improve Growth, Viability and Colonization of Probiotic Microorganisms
}

\section{Pasquale Russo $^{1,2}$, Paloma López ${ }^{3}$, Vittorio Capozzi ${ }^{1,2}$, Pilar Fernández de Palencia ${ }^{3}$, María Teresa Dueñas ${ }^{4}$, Giuseppe Spano ${ }^{2, *}$ and Daniela Fiocco 5}

1 Promis Biotech, Faculty of Agriculture, University of Foggia, Via Napoli 25, 71122 Foggia, Italy; E-Mails: p.russo@unifg.it (P.R.); vittorio.capozzi@gmail.com (V.C.)

2 Department of Food Sciences, Faculty of Agriculture, University of Foggia, Via Napoli 25, 71122 Foggia, Italy

3 Biology Research Center, Department of Molecular Microbiology and Infection Biology, 28040 Madrid, Spain; E-Mails: plg@cib.csic.es (P.L.); pfpalencia@cib.csic.es (P.F.P.)

4 Department of Applied Chemistry, University of Basque Country (UPV/EHU), Paseo Manuel de Lardizábal 3, 20018 Donostia, Spain; E-Mail: mariateresa.duenas@ehu.es

5 Department of Biomedical Science, Faculty of Medicine and Surgery, University of Foggia, Via Pinto, 1, 71122 Foggia, Italy; E-Mail: d.fiocco@unifg.it

* Author to whom correspondence should be addressed; E-Mail: g.spano@unifg.it; Tel.: +39-0-881-589-303; Fax: +39-0-881-589-308.

Received: 16 April 2012; in revised form: 10 May 2012 / Accepted: 11 May 2012 / Published: 18 May 2012

\begin{abstract}
Probiotics, prebiotics and synbiotics are frequently-used components for the elaboration of functional food. Currently, most of the commercialized probiotics are limited to a few strains of the genera Bifidobacteria, Lactobacillus and Streptococcus, most of which produce exopolysaccharides (EPS). This suggests that the beneficial properties of these microorganisms may be related to the biological activities of these biopolymers. In this work we report that a 2 -substituted-(1,3)- $\beta$-D-glucan of non-dairy bacterial origin has a prebiotic effect on three probiotic strains. Moreover, the presence of this $\beta$-D-glucan potentiates in vitro adhesion of the probiotic Lactobacillus plantarum WCFS1 to human intestinal epithelial cells.
\end{abstract}

Keywords: $\beta$-glucans; probiotics; prebiotics; Lactobacillus plantarum 


\section{Introduction}

Probiotics, prebiotics and synbiotics (a mixture of pro- and prebiotics) are today the most frequent components used for the elaboration of functional foods [1]. Probiotics are viable microorganisms able to reach the intestine in an active state and thereby exert positive health effects [2]. A prebiotic is a selectively fermented ingredient that promotes specific changes in the composition and/or activity of the gastrointestinal microbiota which, in turn, confers benefits on host well-being and health [3-5]. Non-digestible oligosaccharides (NDO) fulfill all the criteria for classification as prebiotics. Specifically, the bifidogenic NDO inulin, its hydrolysis product oligofructose, (trans)-galactooligosaccharides and lactulose are the prototype of prebiotic saccharides. Moreover, polysaccharides themselves, or as a source of NDO, are considered potential prebiotics. Bacteria can synthesize, and secrete through the cell wall, polysaccharide layers on their surface, which, together with a few glycoproteins, constitute the glycocalyx. These exocellular polymers comprise the capsular polysaccharides, which form a cohesive layer or capsule covalently linked to the cell surface, and the exopolysaccharides (EPS), which form a slime layer loosely attached to the cell surface or are secreted as free biopolymers into the environment.

Some EPS are important virulence determinants of pathogens, whilst others provide the bases for biotechnological applications [6]. Some EPS have been approved as food additives by the US Food and Drug Administration: the xanthan produced by Xanthomonas campestris, the gellan gum produced by Sphingomonas paucimobilis and the curdlan produced by Agrobacterium.

Lactic acid bacteria (LAB) are able to produce hetero- or homo-exopolysaccharides [7] and these EPS play an important role in the rheology, texture, and mouthfeel of fermented milks and other fermented products. In addition, health benefits have been claimed for EPS from LAB because of their putative antitumoral, immunostimulatory and blood cholesterol lowering activities [8].

Among EPS-producing LAB, Pediococcus parvulus 2.6, isolated from a ropy cider, produces a $(1 \rightarrow 3)$ - $\beta$-D-glucan homopolysaccharide with $50 \%$ of substitutions at positions $O-2$ by side chains composed of a single residue of glucose (2-substituted (1,3)- $\beta$-D-glucan) and with a molecular mass higher than $10^{6} \mathrm{Da}$ [9]. Analysis of the rheological properties of this $\beta$-glucan showed that it has potential utility as a biothickener [10]. The $\beta$-glucan produced by $P$. parvulus 2.6 belongs to a group of linear and branched polysaccharides, the $(1,3)-\beta$-D-glucans, which are produced by several bacteria such as EPS, and are also found in fungi, cereals, and algae [11]. (1,3)- $\beta$-glucans are considered as biological response modifiers, and are attracting attention from the pharmaceutical and functional food industries because of their beneficial effects on human and animal health. Their biological effects are influenced by their degree of branching, molecular mass and tertiary structure [12]. Production of $\beta$-glucans confers to Pediococcus and Lactobacillus strains either an increased adherence to Caco-2 human enterocytes or a macrophage-immunomodulatory capacity [13,14]. Moreover, synthesis of the P. parvulus $2.6 \beta$-glucan confers to Lactobacillus paracasei NFBC 338 higher survival during gastrointestinal passage or technological process [15]. Finally, the production of oat-based products, yogurt and various beverages using the 2 -substituted (1,3)- $\beta$-D-glucan-producing LAB, significantly increased the techno-functional properties of these strains [16].

Human consumption of a ropy, oat-based product, co-fermented with the P. parvulus 2.6 strain, reduced serum cholesterol levels in humans, over and above the effect previously verified for the 
4-substituted (1,3)- $\beta$-D-glucan from oat [17], suggesting a potential application of $(1,3)-\beta$-glucan-producing LAB for the elaboration of functional food.

Prebiotic effects of several EPS produced by LAB have been also reported [18], and availability of prebiotics specifically targeted at the probiotics strains could enable the development of symbiotic versions with enhanced survival in the gut [19].

In order to extend our knowledge on the prebiotic effect of EPS from microbial origin, we evaluated the effect of purified 2-substituted (1,3)- $\beta$-D-glucan on the growth of three probiotic strains, including a recombinant Lactobacillus plantarum strain that over-expresses a $\beta$-glycosidase enzyme. The influence of EPS on the capability of L. plantarum to adhere to human intestinal cells was also investigated. The results reported in this paper provide additional information on the positive effect of EPS as prebiotic substrates.

\section{Results and Discussion}

\subsection{Overexpression of a $\beta$-Glycosidase Gene in L. plantarum WCFS1}

Since EPS are macromolecules that cannot cross the cell membrane through the common transport systems, they first must be hydrolyzed to be metabolized in the cells. Glycosidases (EC 3.2.1) are enzymes that hydrolyze the glycosidic bonds of polysaccharides, hence facilitating the release of the smaller sugars involved in nutrient acquisition. Therefore, we have determined whether an increased glycosidase activity could augment any prebiotic character of the 2 -substituted (1,3)- $\beta$-D-glucan synthesized by $P$. parvulus 2.6. Therefore, the $\beta$-glycosidase (bgl, lp_3629) gene from L. plantarum strain WCFS1, which was previously characterized in response to abiotic stress [20], was cloned into the pGIZ906 vector and overexpressed in L. plantarum. Overproduction of Bgl in the recombinant strain (named L. plantarum WCFS1 $\beta$-gal) was confirmed by SDS-PAGE. Figure 1 shows that this strain produced large amounts of a $53 \mathrm{kDa}$ protein, corresponding to the theoretical molecular mass of Bgl. Acebrón et al. [21] demonstrated biochemically that the lp_3629 gene encodes a functional $\beta$-galactosidase (EC 3.2.1.23). However, the amino acid sequence of Bgl shows that it belongs to the glycosyl hydrolase family 1 . Enzymes in this family have a broad substrate specificity [22]. Therefore, the glucosidic bonds of the P. parvulus $2.6 \beta$-glucan could be hydrolyzed by Bgl.

\subsection{Prebiotic Characterization of EPS}

Furthermore, we analyzed the potential prebiotic effect of EPS from bacterial origin on the survival of three strains belonging to the Lactobacillus genus: L. plantarum WCFS1, the isogenic recombinant L. plantarum WCFS1 1 -gal and Lactobacillus acidophilus strain NCFM. These microorganisms are known for their beneficial contribution to human health and are widely distributed in fermented food [2,23,24].

The hypothetical role of the 2-substituted (1,3)- $\beta$-glucan produced by $P$. parvulus 2.6 as a sugar source for bacterial metabolism was evaluated by testing for microbial growth in a chemically defined medium which has been recently described [25]. To confirm that a source of carbohydrate was essential for bacterial development, the medium was initially prepared omitting D-glucose. Under this condition, no growth was detected for any of the strains examined (data not shown), in agreement with Terrade and Mira de Orduña [26], who did not observe biomass yield in the same medium without D-ribose as 
a unique sugar substrate. When only D-glucose was added to the medium as a sugar source, both L. plantarum WCFS1 and L. plantarum WCFS1 1 -gal strains showed a very similar growth kinetics, reaching the stationary phase after approximately $24 \mathrm{~h}\left(\mathrm{OD}_{600}=2.00\right)$ (Figure 2). In contrast, L. acidophilus exhibited a much lower growth at all the monitored times. The maximum cell concentration was reached after $24 \mathrm{~h}\left(\mathrm{OD}_{600}=0.75\right)$, followed by a death-phase after $30 \mathrm{~h}\left(\mathrm{OD}_{600}=0.65\right)$ (Figure 2$)$, as even confirmed by plate count analysis (data not shown). Since the medium used in this work was optimized to analyze LAB isolated from wine [25], the reduced growth observed for L. acidophilus may be related to the composition of the growth media.

Figure 1. Sodium dodecylsulphate-polyacrylamide gel electrophoresis (SDS-PAGE) profiles of total protein extracts from L. plantarum WCFS1 harboring pGIZ906 (lane A) or the recombinant PldhL-bgl-pGIZ906 (strain WCFS1 $\beta$-gal, lane B). The molecular size was determined by comparison with a protein ladder (M). M: prestained SDS-PAGE standards, low range. Molecular mass of the marker bands is indicated in $\mathrm{kDa}$.

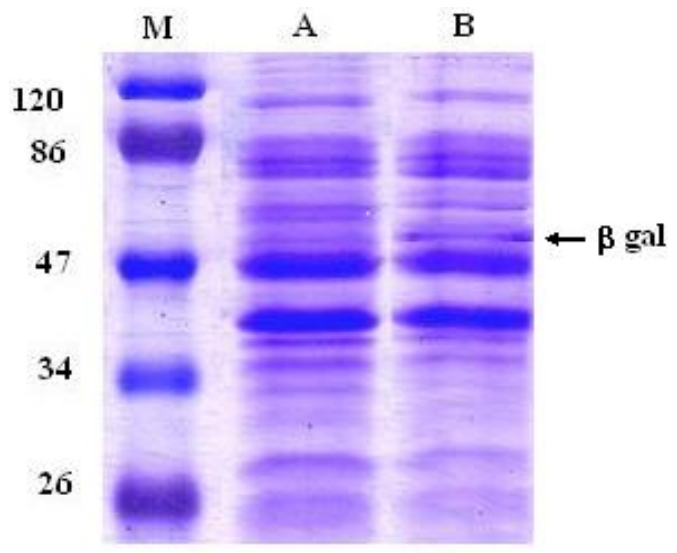

Figure 2. Kinetics of growth of L. plantarum WCFS1 (blue line), L. plantarum WCFS1 $\beta$-gal (red line) and L. acidophilus (green line) in a chemically defined medium (CDM) supplemented with D-glucose $\left(10 \mathrm{~g} \cdot \mathrm{L}^{-1}\right)$. Growth was monitored during $30 \mathrm{~h}$ by spectrophotometric determination of the optical density at $600 \mathrm{~nm}\left(\mathrm{OD}_{600}\right)$.

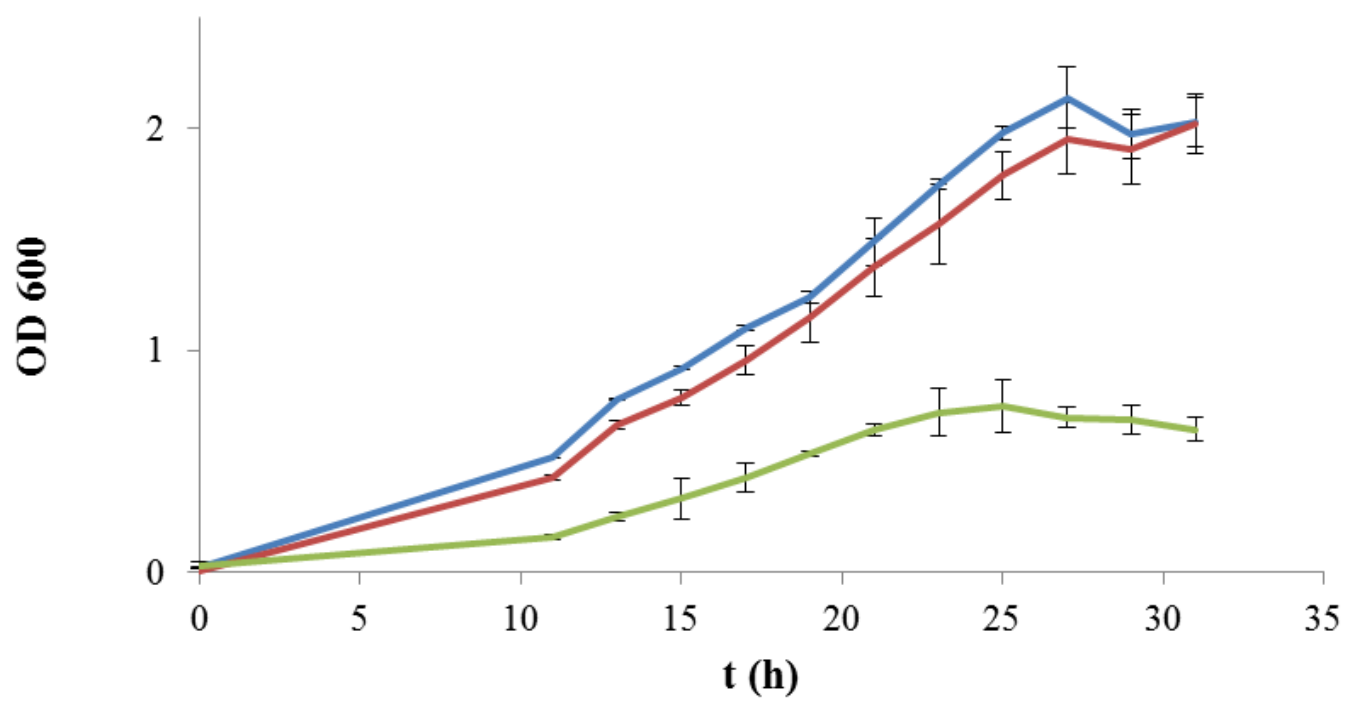


The exopolysaccharide used in this study was obtained from $P$. parvulus 2.6 and the yield of purified EPS was about $200 \mathrm{mg}$ per liter of microbial culture, as previously reported by Garai-Ibabe et al. [14]. Therefore, due to their low availability, the experimental assays in presence of EPS were performed in microtitre plates. The concentration of viable cells, expressed as colony forming units (cfu), was determined at incubation times $0,3,6,9,24,27$, and $30 \mathrm{~h}$ (Figure 3). In the L. plantarum strains analyzed (Figure 3A,B), a significant difference of more than 1-log was observed when bacteria were grown in a medium supplemented with only D-glucose or with EPS as carbon source. In addition, a beneficial effect was also observed in the viability of the two strains when inoculated in medium containing D-glucose, supplemented with EPS. In particular, L. plantarum WCFS1 $1 \beta$-gal showed an increased biomass in all phases when both substrates (D-glucose and EPS)

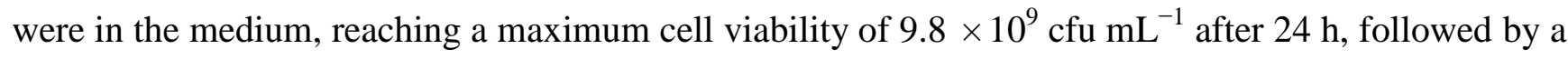
subsequent slight decrease (Figure 3B). A similar pattern of growth was observed in presence of only glucose, but a reduced viability was detected in the logarithmic and stationary phase, with a maximum concentration corresponding to $7.7 \times 10^{9} \mathrm{cfu} \mathrm{mL}^{-1}$ after $24 \mathrm{~h}$ (Figure 3B). In contrast, L. plantarum WCFS1 showed an identical overlapping of the viability during the first $24 \mathrm{~h}$ (approximately $6.0 \times 10^{9} \mathrm{cfu} \mathrm{mL}^{-1}$ ), independently from the presence or absence of EPS in addition to D-glucose. Interestingly, after the critical time of $24 \mathrm{~h}$ of incubation, bacteria where still able to grow in EPS-containing media (around $9.0 \times 10^{9} \mathrm{cfu} \mathrm{mL}^{-1}$, at $30 \mathrm{~h}$ ), while their number gradually declined in media supplemented with only D-glucose $\left(5.4 \times 10^{9} \mathrm{cfu} \mathrm{mL}^{-1}\right.$, at $\left.30 \mathrm{~h}\right)$ (Figure 3A).

Figure 3. Growth kinetics of L. plantarum WCFS1 (A), L. plantarum WCFS1 $\beta$-gal (B) and $L$. acidophilus $(\mathbf{C})$ in a chemically defined medium supplemented with D-glucose $\left(10 \mathrm{~g} \mathrm{~L}^{-1}\right)$ (blue line), exopolysaccharides (EPS) $(0.5 \% \mathrm{w} / \mathrm{v})$ (green line) or both (red line). Values of growth in presence of only EPS are reported on a secondary $Y$-axis. Growth was monitored over a $30 \mathrm{~h}$ period, by colony forming units (cfu) counting.

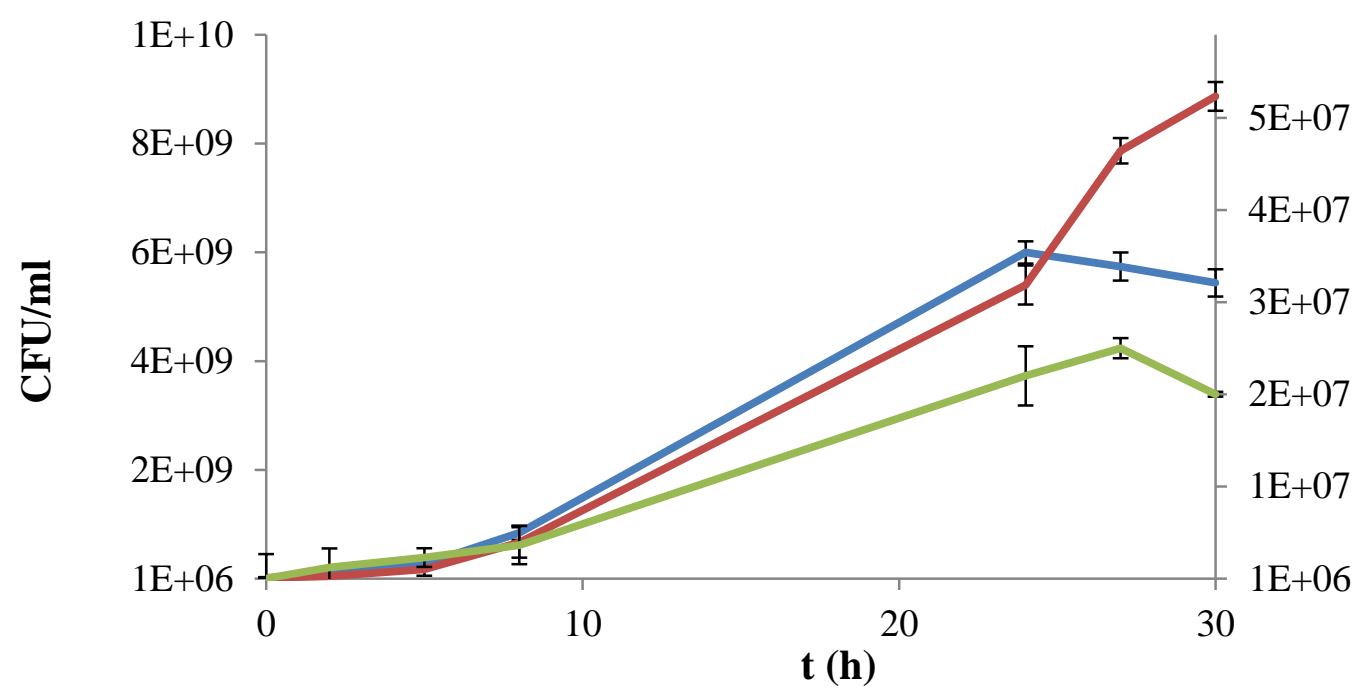

(A) 
Figure 3. Cont.

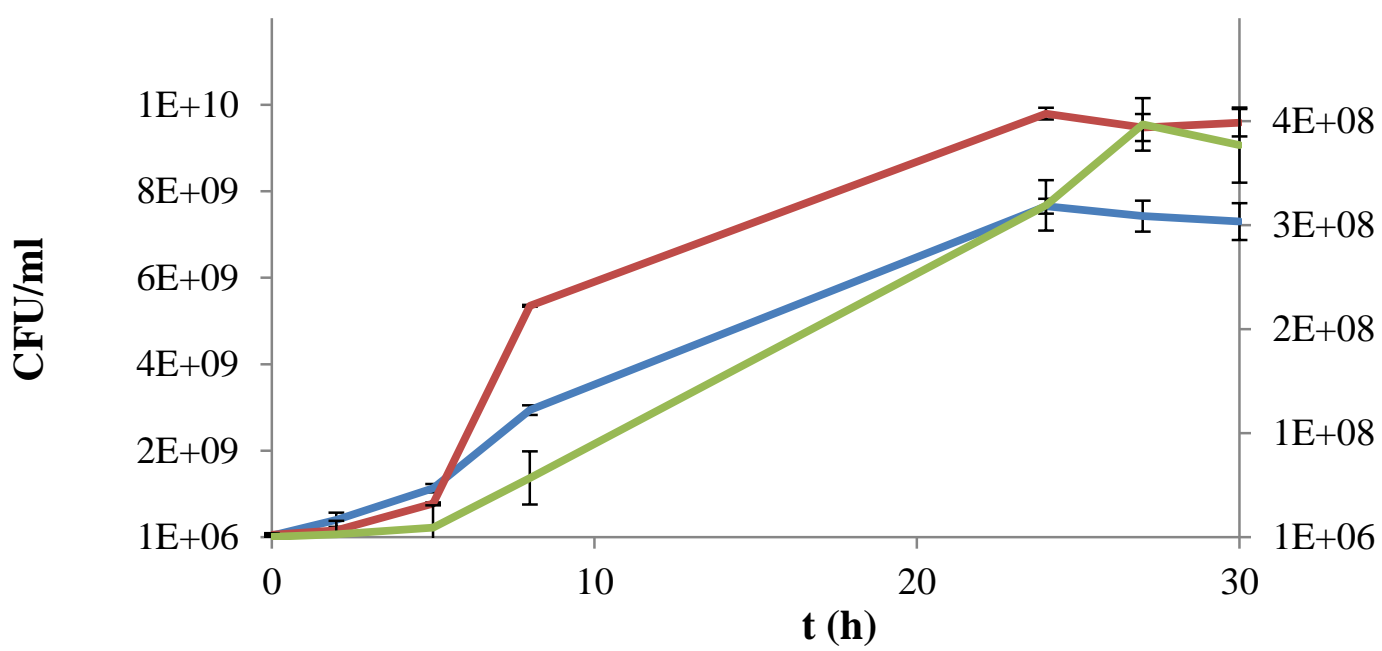

(B)

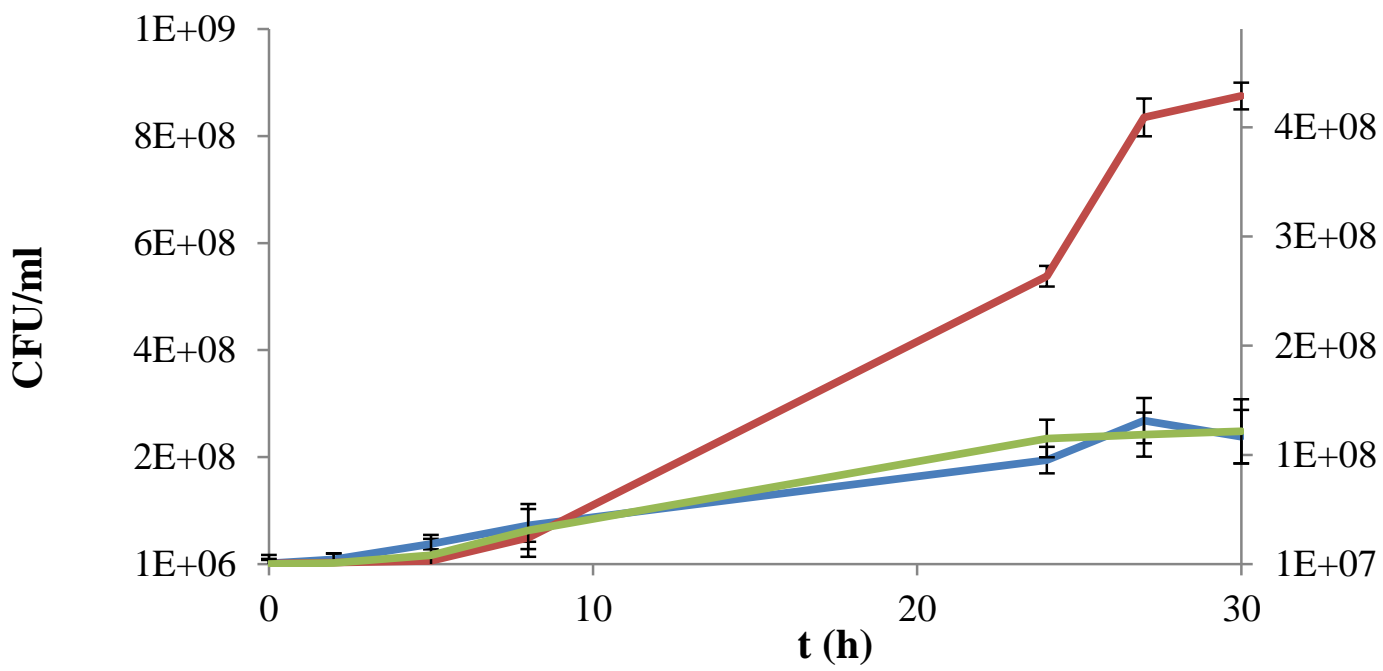

(C)

The growth rate observed for L. acidophilus in the presence of only EPS was lower compared to the assay carried out when only D-glucose was provided, with plate counts of 1.3 and $2.3 \times 10^{8} \mathrm{cfu} \mathrm{mL}^{-1}$, as maximum viable cell concentration, respectively (Figure 3C). Surprisingly, the availability of both substrates stimulated the growth of L. acidophilus by delaying its entry into stationary phase. Indeed, after $24 \mathrm{~h}$ the cfu were 2.5 -fold more copious than in the other conditions, and at the last experimental time a value of $8.7 \times 10^{8} \mathrm{cfu} \mathrm{mL}^{-1}$ was reached (Figure $3 \mathrm{C}$ ).

The results reported in this study revealed a positive effect of the $\beta$-D-glucan on the growth of the investigated strains, suggesting that its use as a prebiotic may positively modulate the growth of probiotic organisms. In particular, the availability of both D-glucose and EPS extended the logarithmic phase for both L. plantarum WCFS1 and L. acidophilus strains. To explain this result, we assume that after the immediate consumption of D-glucose, bacteria could use EPS as a further carbon source. The release of hydrolytic enzymes under conditions of starvation, or the lysis of bacteria occurring at late exponential or stationary-phase, would enhance the activity of glycosidases in the extracellular environment. It is well known that the microbial species investigated in this study have a genetic makeup that allows them to use a variety of carbohydrate sources. Indeed, in silico analysis of the L. plantarum 
WCFS1 genome predicted 25 complete phosphoenolpyruvate sugar-transferase systems (PTS) enzyme II complexes, several incomplete complexes and 30 transporter systems that are involved in the transport of carbon sources [27]; likewise, the genome of L. acidophilus encodes a large variety of genes related to carbohydrate utilization, including 20 PTS and five transporters of the ABC family [24]. In addition, the substrate specificity cannot be predicted for some PTS and other carbon-uptake systems, and various sugar transport systems are known to import more than one substrate, thereby expanding the carbon transport capacity of these species even further [27].

In the medium supplemented with only EPS, we always observed an increase in the number of viable cells after $30 \mathrm{~h}$ of incubation, corresponding to approximately $1 \log$ for L. acidophilus and L. plantarum WCFS1, while for the engineered L. plantarum WCFS1 $\beta$-gal strain, viable bacteria numbers increased from $1.3 \times 10^{6} \mathrm{cfu} \mathrm{mL}^{-1}$ to $3.8 \times 10^{8} \mathrm{cfu} \mathrm{mL}^{-1}$. In addition an efficient co-metabolism of glucose and EPS was observed for this strain, which resulted in an increase of cell viability. This confirms that the overexpression of the $1 p \_3629$ gene encoding a functional $\beta$-glycosidase [21] could lead to a more marked hydrolysis of EPS, thus enhancing bacterial survival. Expression of similar enzymes might be a widespread strategy of the gut synbiotic bacteria. Indeed, in order to survive in the lower intestinal tract, bifidobacteria produce various kinds of exo- and endoglycosidases in surface-bound and/or extracellular forms, by which they can utilize diverse carbohydrates [28]. Recent genome sequence analysis of the probiotic Bifidobacterium longum NCC2705 revealed that more than $8.5 \%$ of the total predicted proteins were involved in the degradation of oligo- and polysaccharides, suggesting a superior ability of this organism to adapt to the poor nutritional conditions of the low intestine [29].

We also found that the growth of L. plantarum WCFS1 and L. acidophilus was favored by the simultaneous availability of both substrates, suggesting a synergistic effect of EPS and D-glucose in promoting bacterial viability. However, further studies are necessary to explain the biochemical and molecular bases of these observations.

Because EPS are known to be involved in biofilm formation [30], several authors have investigated in vitro the link between EPS production and the capability to colonize the intestinal environment $[13,14]$. However, such studies did not contemplate the influence that exogenous EPS could have on the adhesion level of other microbial species. Therefore, in this work we investigated the effect of the 2 -substituted (1-3)- $\beta$-D-glucan on the adhesive ability to human intestinal epithelial cells of L. plantarum WCFS1, a strain that does not show any mucous production in culture medium supernatants. In the absence of the purified EPS, approximately $4 \%$ of the bacteria adhered to Caco- 2 cells. This is consistent with previous work reporting a percentage of adhesion ranging from $2 \%$ to $10 \%$ for commercial probiotic strains [31]. However, when L. plantarum was incubated with $0.5 \% \mathrm{w} / v$ of EPS, the percentage of bound bacteria increased approximately five-fold (Figure 4). It has already been demonstrated that prebiotics such as oligosaccharides can influence adhesion of specific strains [32]. Intriguingly, to the best of our knowledge, no global positive effects of these molecules on microbial adhesion potential has been detected [32-35]. 
Figure 4. Percentage of adhesion of L. plantarum WCFS1 to Caco-2 cells after incubation with, or without EPS $(0.5 \% w / v)$.

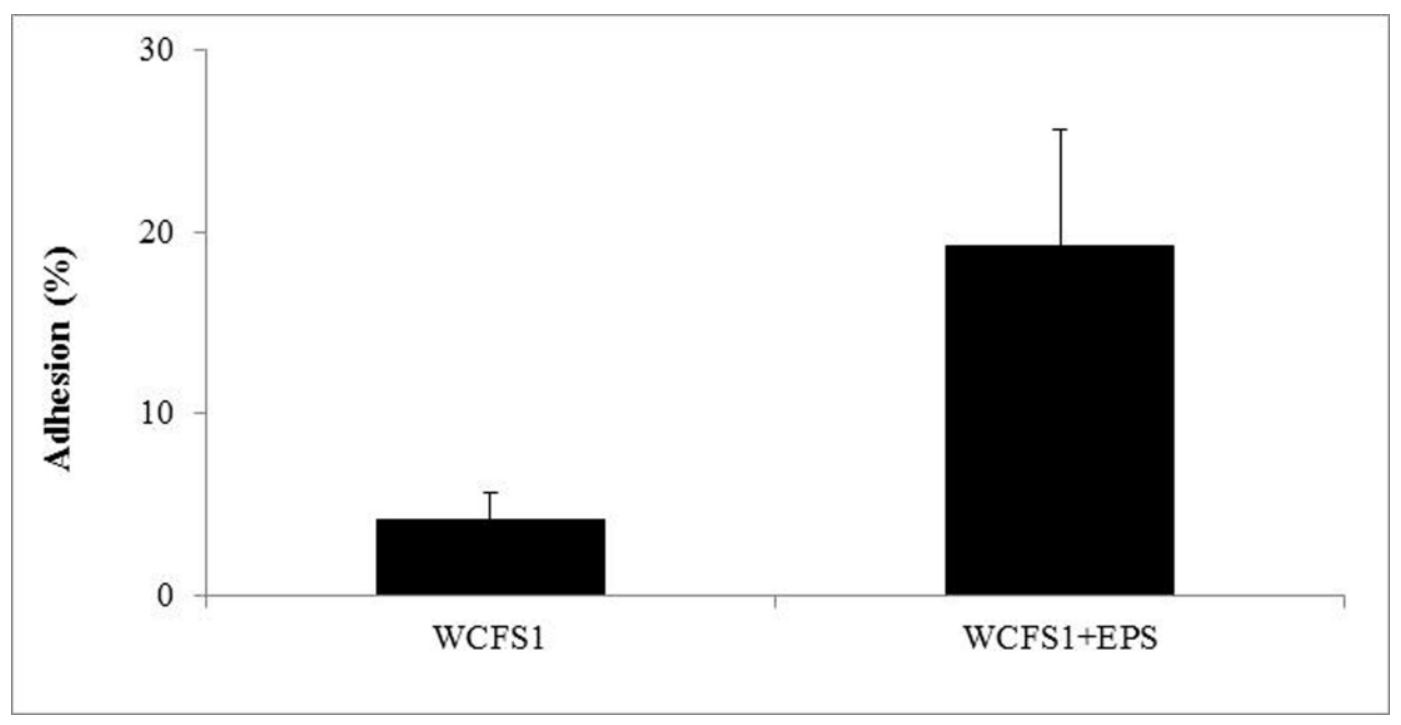

As components of the bacterial cell surface, the EPS produced and secreted by LAB are likely to underlie inter-cellular recognition mechanisms and/or the process of biofilm formation. In this regard, $\beta$-D-glucans might promote the initial steps of adhesion to host intestinal cells. This finding raises considerable interest for its potential applications in the design of symbiotic food products. Indeed, we can speculate that addition of specific EPS in the food matrix that vehicles probiotics might enhance microbial adhesion to the intestinal epithelium, thus improving gut colonization by beneficial microorganisms. A persistent or at least transient colonization of the gut is a desirable feature of probiotics, as a close and prolonged interaction with the host allows them to accomplish beneficial effects such as immune function modulation and pathogen exclusion [36]. Moreover, as observed for other prebiotic NDO, $\beta$-D-glucans might also contrast pathogen adhesion to the intestinal mucosa and/or act as antioxidants to protect probiotics from the intestinal hostile environment [37]. Further research shall be undertaken to address these issues and test such hypothesis.

\section{Experimental Section}

\subsection{Bacterial Strains and Media}

The bacterial strains used in this study were L. plantarum strain WCFS1 [27], its recombinant isogenic strain that overexpresses the $b g l$ gene encoding a $\beta$-galactosidase ( $L$. plantarum WCFS1 $\beta$-gal) and the L. acidophilus strain NCFM [24,38].

A chemically defined medium was prepared as reported by Terrade et al. [25] but without D-ribose and modifying the final $\mathrm{pH}$ at 6.2. The growth kinetics was evaluated either in absence of any carbon source or supplementing the same medium with D-glucose $\left(10 \mathrm{~g} \cdot \mathrm{L}^{-1}\right)$, EPS $(0.05 \% w / v)$ or both. The recombinant strain was grown in media that included $10 \mu \mathrm{g} \cdot \mathrm{mL}^{-1}$ of erythromycin. L. plantarum strains and L. acidophilus were always incubated without shaking at the optimal temperature of $30{ }^{\circ} \mathrm{C}$ and $37{ }^{\circ} \mathrm{C}$, respectively. 
P. parvulus 2.6 [9] was isolated from ropy cider at the Department of Applied Chemistry, Faculty of Chemistry (University of the Basque Country UPV/EHU, San Sebastián, Spain).

Strains were kept in de Man Rogosa Sharpe (MRS) broth (Pronadisa, Madrid, Spain) supplemented with $20 \%(v / v)$ glycerol for long-term storage at $-80{ }^{\circ} \mathrm{C}$.

\subsection{Production of EPS by P. parvulus 2.6}

To obtain a pre-inocula for EPS production, $P$. parvulus 2.6 was grown on MRS broth, supplemented with $0.05 \%(w / v)$ L-cysteine hydrochloride (Merck, Darmstad, Germany) and $0.1 \%(w / v)$ Tween 80 (Pronadisa, Barcelona, Spain). For production of the EPS, P. parvulus 2.6 was grown in the semi-defined media MST [39], containing glucose $\left(50 \mathrm{~g} \cdot \mathrm{L}^{-1}\right)$ and ethanol 4.9\% (w/v), in a 3-L fermenter (Bioflo 110, New Brunswick Scientific, Enfield, CT), at $30{ }^{\circ} \mathrm{C}$ for $96 \mathrm{~h}$. The $\mathrm{pH}$ was controlled at 5.2 with $5 \mathrm{~N}$ $\mathrm{NaOH}$, the agitation was kept at 50-70 rpm to keep the fermentation broth homogeneous, and nitrogen gas $\left(0.2 \mathrm{~L} \cdot \mathrm{h}^{-1}\right)$ was sparged through the headspace continuously to maintain anaerobic conditions.

Bacterial cells were removed from fermented media by centrifugation $\left(16,000 \times g, 4{ }^{\circ} \mathrm{C}, 30 \mathrm{~min}\right)$ after $96 \mathrm{~h}$ of culture. The clear supernatant was collected and the EPS precipitated by adding three volumes of cold absolute ethanol, and maintained overnight at $4{ }^{\circ} \mathrm{C}$. The precipitate was recovered by centrifugation at $14,000 \times g$ for $10 \mathrm{~min}$ at $4{ }^{\circ} \mathrm{C}$, the resulting EPS pellet was dissolved in deionized water, and the EPS was recovered by three cycles of precipitation with ethanol. The final precipitate was dissolved in and dialyzed against deionized water, using a dialysis membrane (Medicell International, Ltd., London, U.K.) having a cut-off of $3.5 \mathrm{kDa}$, for 2-3 days (water changed twice each day). After dialysis, the precipitate was lyophilized.

For the experimental assay, media were prepared by adding appropriate volumes of a $100 \times$ concentrated stock solution of EPS to obtain the desired final concentration.

\subsection{Overexpression of Bgl Gene in L. plantarum}

All the plasmid constructions were performed in Escherichia coli TG1 strain. The bgl gene was cloned in the pGIZ906 vector, a pLAB1301-derivative with a $0.352-\mathrm{kb}$ insert containing the $l d h L$ gene expression signals of $L$. plantarum WCFS1 [27,40]. The $b g l$ gene was amplified from $L$. plantarum WCFS1 genomic DNA using forward (5'-AAAACTGCAGGAGTTCCGGAAGGCTTT-3') and reverse (5'-GCTCTAGATCAAAACCCATTCCGTTCCCCA-3') primers, harbouring PstiI and XbaI sites (underlined sequence), respectively. The PCR fragment was digested with PstI-XbaI and located between the NsiI-XbaI sites of pGIZ906 vector by ligation to generate a PldhL-bgl transcriptional fusion. The recombinant plasmid was electroporated into L. plantarum WCFS1 and transformants were identified on MRS plates containing erythromycin. Plasmid DNA preparation was performed following standard procedures. The absence of mutations in the plasmidic $b g l$ gene was confirmed by DNA sequencing. 


\subsection{Protein Extraction and SDS-PAGE}

Cells of $L$. plantarum $\beta$-gal grown in $30 \mathrm{~mL}$ of MRS to middle exponential phase were sedimented by centrifugation $\left(5000 \times \mathrm{g}, 10 \mathrm{~min}, 4{ }^{\circ} \mathrm{C}\right.$ ) and washed twice with $0.1 \mathrm{M}$ sodium phosphate buffer $\mathrm{pH}$ 7.0. Bacteria were resuspended in $600 \mu \mathrm{L}$ of cold phosphate buffer containing $0.1 \mathrm{mM}$ phenylmethylsulfonyl fluoride (PMSF) (Roche Applied Science, Indianapolis, IN), mixed with glass beads (212-300 $\mu \mathrm{m} \varnothing$, Biospec Products, Bartlesville, OK) and mechanically disrupted in a Mini Beadbeater ${ }^{\mathrm{TM}}$ (Biospec Products) (four cycles of $1 \mathrm{~min}$ at max speed followed by $1 \mathrm{~min}$ on ice). After removal of cellular debris by centrifugation $\left(13,000 \times \mathrm{g}, 10 \mathrm{~min}, 4{ }^{\circ} \mathrm{C}\right)$, supernatants were aliquoted and stored at $-20^{\circ} \mathrm{C}$. Quantification of total protein extracts was determined with a Bio-Rad Protein Assay (Biorad, Milan, Italy), according to the manufacturer's instructions. Protein fractionation was carried out by sodium dodecylsulphate-polyacrylamide gel electrophoresis (SDS-PAGE) at $150 \mathrm{~V}$ for $1 \mathrm{~h}$ in a $12 \%(w / v)$ resolving and $4 \%(w / v)$ stacking gel. A prestained SDS-PAGE standards, low range (Biorad) was used as protein marker. After PAGE, protein bands were revealed by staining with coomassie brilliant blue (0.2\% CBB R-250).

\subsection{Kinetics of Cell Growth with Different Carbon Sources}

Strains from glycerol stocks were inoculated into MRS medium and grown to middle exponential phase $\left(\mathrm{OD}_{600}=0.8\right.$ and for $L$. plantarum and $\mathrm{OD}_{600}=0.6$ for $L$. acidophilus strains $)$. These cultures were used as inocula (dilution 1:100 in fresh defined medium), after harvest by centrifugation $(5000 \times g$, $5 \mathrm{~min}$ ), two washes and resuspension in the corresponding chemically defined medium. The kinetics of bacterial growth were spectrophotometrically monitored during $24 \mathrm{~h}$ in media supplemented or not with D-glucose. The analysis was performed in the wells of microtitre plates containing $200 \mu \mathrm{L}$ of chemically defined medium in the presence of only glucose, only EPS or both. The growth of all strains was analyzed by determination of colony forming units (cfu) by plate count of samples incubated for $30 \mathrm{~h}$ at optimal temperature. Experiments were performed in triplicate.

\subsection{Adhesion Assay}

Adhesion assays were performed using the Caco-2 epithelial cell line, originated from human colonic carcinoma, and kindly gifted by Dr. C. Lamacchia, University of Foggia. Caco-2 cells were grown as previously described [14,31,41] and supplemented with $2 \mathrm{mM}$ L-glutamine (Sigma-Aldrich, St. Louis, MO). In post-confluent cultures, the viable cell number, as counted in a Burker chamber, was about $4.3 \times 10^{4}$ cells per well and bacteria were added to achieve a final concentration of approximately $4.3 \times 10^{8} \mathrm{cfu} / \mathrm{mL}$ (ratio 1000:1 bacteria to Caco-2 cells). Adhesion experiments were then performed according to Bove et al. [41]. Serial dilutions of the samples were plated onto MRS-agar plates to determine the number of cell-associated bacteria (viable counts) expressed as cfu. The adhesion percentage was calculated by comparing the number of cfu from washed wells (cell-bound bacteria) with those from control unwashed wells (unbound and bound bacteria). Experiments were performed in triplicate. 


\subsection{Statiscal Analysis}

Significant differences between averages of duplicate measurements were evaluated by performing $t$-tests after analysis of variance at a confidence level of $p=0.05$.

\section{Conclusions}

In this work we reported the prebiotic action of a 2 -substituted (1-3)- $\beta$-D-glucan of bacterial origin on three probiotic LAB. In particular, our results showed that all the investigated bacterial strains were able to use EPS as a substrate for their growth. In addition, the simultaneous availability of D-glucose and EPS as glucid sources delayed the entry into stationary phase of L. plantarum WCFS1 $\beta$-gal and L. acidophilus, as well as encouraged the growth of L. plantarum WCFS1 and L. acidophilus in the exponential-phase, thus suggesting their synergistic effect in promoting metabolic processes. The pronounced rate of growth observed for the recombinant strain L. plantarum WCFS1 $\beta$-gal confirms the positive influence of glycosidases enzymes in increasing the prebiotic potential of EPS. Notably, this $\beta$-D-glucan increases the binding of the non-ropy L. plantarum WCFS1 strain to intestinal cells. These results suggest that mixed probiotic LAB strains, able either to produce or consume $\beta$-glucans, could be a suitable strategy for the development of new types of functional food.

\section{Acknowledgments}

This work was funded by the Italian Ministry for the Development in the framework of the projects named "Industria 2015, Bando Nuove Tecnologie per il Made in Italy_Realizzazione di una innovativa pasta alimentare funzionale arricchita di componenti bioattivi e probiotici" and "Pro. Ali.Fun." as well as by the Spanish Ministery of Innovation and Science grant AGL2009-12998-C03. We would like to thank M. Kleerebezem for providing the L. plantarum WCFS1 strain and Stephen Elson for the critical reading of the manuscript.

\section{Conflict of Interest}

The authors declare no conflict of interest.

\section{References}

1. Reid, G.; Sanders, M.E.; Gaskins, H.R.; Gibson, G.R.; Mercenier, A.; Rastall, R.; Roberfroid, M.; Rowland, I.; Cherbut, C.; Klaenhammer, T.R. New scientific paradigms for probiotics and prebiotics. J. Clin. Gastroenterol. 2003, 37, 105-118.

2. De Vrese, M.; Schrezenmeir, J. Probiotics, prebiotics, and synbiotics. Adv. Biochem. Eng. Biotechnol. 2008, 111, 1-66.

3. Cummings, J.H.; Macfarlane, G.T.; Englyst, H.N. Prebiotic digestion and fermentation. Am. J. Clin. Nutr. 2001, 73, 415-420.

4. Marteau, P.R.; de Vrese, M.; Cellier, C.J.; Schrezenmeir, J. Protection from gastrointestinal diseases with the use of probiotics. Am. J. Clin. Nutr. 2001, 73, 430-436. 
5. Gibson, G.R.; Probert, H.M.; van loo, J.; Rastall, R.A.; Roberfroid, M. Dietary modulation of the human colonic microbiota: Updating the concept of prebiotics. Nutr. Res. Rev. 2004, 17, 259-275.

6. Sutherland, I.W. Microbial polysaccharides from Gram-negative bacteria. Int. Dairy J. 2001, 11, 663-674.

7. Ruas-Madiedo, P.; Moreno, J.A.; Salazar, N.; Delgado, S.; Mayo, B.; Margolles, A.; de los Reyes-Gavilán, C.G. Screening of exopolysaccharide-producing lactobacillus and bifidobacterium strains isolated from the human intestinal microbiota. Appl. Environ. Microbiol. 2007, 73, 4385-4388.

8. Liu, C.-F.; Tseng, K.-C.; Chiang, S.S.; Lee, B.H.; Hsu, W.-H.; Pan, T.-M. Immunomodulatory and antioxidant potential of Lactobacillus exopolysaccharides. J. Sci. Food Agric. 2011, 91, 2284-2291.

9. Dueñas-Chasco, M.T.; Rodríguez-Carvajal, M.A.; Mateo, P.T.; Franco-Rodríguez, G.; Espartero, J.; Irastorza-Iribas, A.; Gil-Serrano, A.M. Structural analysis of the exopolysaccharide produced by Pediococcus damnosus 2.6. Carbohydr. Res. 1997, 303, 453-458.

10. Velasco, S.E.; Areizaga, J.; Irastorza, A.; Dueñas, M.T.; Santamaría, A.; Muñoz, M.E. Chemical and rheological properties of the $\beta$-glucan produced by Pediococcus parvulus 2.6. J. Agric. Food Chem. 2009, 57, 1827-1834.

11. Zekovic, D.B.; Kwiatkowski, S.; Vrvic, M.M.; Jakovljevic, D.; Moran, C.A. Natural and modified $(1 \rightarrow 3)-\beta$-D-glucans in health promotion and disease alleviation. Crit. Rev. Biotechnol. 2005, 25, 205-230.

12. Sletmoen, M.; Stokke, B.T. Higher order structure of (1,3)- $\beta$-D-glucans and its influence on their biological activities and complexation abilities. Biopolymers 2008, 89, 310-321.

13. Fernández de Palencia, P.; Werning, M.L.; Sierra-Filardi, E.; Dueñas, M.T.; Irastorza, A.; Corbí, A.L.; López, P. Probiotic properties of the 2 -substituted $(1,3)-\beta$-D-glucan-producing bacterium Pediococcus parvulus 2.6. Appl. Environ. Microbiol. 2009, 75, 4887-4891.

14. Garai-Ibabe, G.; Dueñas, M.T.; Irastorza, A.; Sierra-Filardi, E.; Werning, M.L.; López, P.; Corbi, A.L.; Fernández de Palencia, P. Naturally occurring 2-substituted (1,3)- $\beta$-D-glucan producing Lactobacillus suebicus and Pediococcus parvulus strains with potential utility in the production of functional foods. Biores. Technol. 2010, 101, 9254-9263.

15. Stack, H.M.; Kearney, N.; Stanton, C.; Gerald, F.; Fitzgerald, G.F.; Ross, R.P. Association of $\beta$-glucan endogenous production with increased stress tolerance of intestinal lactobacilli. Appl. Environ. Microbiol. 2010, 76, 500-507.

16. Kearney, N.; Stack, H.M.; Tobin, J.; Chaurin, V.; Fenelon, M.A.; Fitzgerald, G.F.; Ross, R.P.; Stanton, C. Lactobacillus paracasei NFBC 338 producing recombinant beta-glucan positively influences the functional properties of yoghurt. Int. Dairy J. 2011, 21, 561-567.

17. Mårtensson, O.; Biörklund, M.; Lambo, M.A.; Dueñas-Chasco, M.T.; Irastorza, A.; Holst, O.; Norin, E.; Walling, G.; Öste, R.; Önning, G. Fermented ropy, oat based products reduce cholesterol levels and stimulate the bifidobacteria flora in humans. Nutr. Res. 2005, 25, 429-442.

18. Korakli, M.; Gänzle, M.G.; Vogel, R.F. Metabolism by bifidobacteria and lactic acid bacteria of polysaccharides from wheat and rye, and exopolysaccharides produced by Lactobacillus sanfranciscensis. J. Appl. Microbiol. 2002, 92, 958-965.

19. Manning, T.S.; Gibson, G.R. Prebiotics. Best Pract. Res. Clin. Gastroenterol. 2004, 18, 287-298. 
20. Spano, G.; Rinaldi, A.; Ugliano, M.; Moio, L.; Beneduce, L.; Massa, S. A $\beta$-glucosidase gene isolated from wine Lactobacillus plantarum is regulated by abiotic stresses. J. Appl. Microbiol. 2005, 98, 855-861.

21. Acebrón, I.; Curiel, J.A.; de las Rivas, B.; Muñoz, R.; Mancheño, J.M. Cloning, production, purification and preliminary crystallographic analysis of a glycosidase from the food lactic acid bacterium Lactobacillus plantarum CECT 748T. Prot. Expr. Purif. 2009, 68, 177-182.

22. Ketudat-Cairns, J.R.; Esen, A. $\beta$-glucosidases. Cell. Mol. Life Sci. 2010, 67, 3389-3405.

23. Molin, G. Probiotics in foods not containing milk or milk constituents, with special reference to Lactobacillus plantarum 299v. Am. J. Clin. Nutr. 2001, 73, 380-385.

24. Altermann, E.; Russell, W.M.; Azcarate-Peril, M.A.; Barrangou, R.; Buck, B.L.; McAuliffe, O.; Souther, N.; Dobson, A.; Duong, T.; Callanan, M.; et al. Complete genome sequence of the probiotic lactic acid bacterium Lactobacillus acidophilus NCFM. Proc. Natl. Acad. Sci. USA 2005, 102, 3906-3912.

25. Terrade, N.; Noël, R.; Couillaud, R.; Mira de Orduña, R. A new chemically defined medium for wine lactic acid bacteria. Food Res. Int. 2009, 42, 363-367.

26. Terrade, N.; Mira de Orduña, R. Determination of the essential nutrient requirements of wine-related bacteria from the genera Oenococcus and Lactobacillus. Int. J. Food Microbiol. 2009, $133,8-13$.

27. Kleerebezem, M.; Boekhorst, J.; van Kranenburg, R.; Molenaar, D.; Kuipers, O.P.; Leer, R.; Tarchini, R.; Peters, S.A.; Sandbrink, H.M.; Fiers, M.W.; et al. Complete genome sequence of Lactobacillus plantarum WCFS1. Proc. Natl. Acad. Sci. USA 2003, 100, 1990-1995.

28. Katayama, T.; Fujita, K.; Yamamoto, K. Novel bifidobacterial glycosidases acting on sugar chains of mucin glycoproteins. J. Biosci. Bioeng. 2005, 99, 457-465.

29. Schell, M.A.; Karmirantzou, M.; Snel, B.; Vilanova, D.; Berger, B.; Pessi, G.; Zwahlen, M.C.; Desiere, F.; Bork, P.; Delley, M.; et al. The genome sequence of Bifidobacterium longum reflects its adaptation to the human gastrointestinal tract. Proc. Natl. Acad. Sci. USA 2002, 99, 14422-14427.

30. Dols-Lafargue, M.; Lee, H.Y.; le marrec, C.; Heyraud, A.; Chambat, G.; Lonvaud-Funel, A. Characterization of gtf, a glucosyltransferase gene in the genomes of Pediococcus parvulus and Oenococcus oeni. Appl. Environ. Microbiol. 2008, 74, 4079-4090.

31. Fernández de Palencia, P.; López, P.; Corbí, A.L.; Peláez, C.; Requena, T. Probiotic strains: Survival under simulated gastrointestinal conditions, in vitro adhesion to Caco-2 cells and effect on cytokine secretion. Eur. Food Res. Technol. 2008, 227, 1475-1484.

32. Van den Abbeele, P.; van de Wiele, T.; Grootaert, C.; Verstraete, W.; Gérard, P.; Bruneau, A.; Rabot, S.; Possemiers, S. Arabinoxylans and Inulin Modulate the Luminal and Mucosa-Associated Bacteria in Vitro and in Vivo. In Dietary Fibre: New Frontiers for Food and Health; van der Kamp, J.W., Jones, J.M., McCleary, B.V., Topping, D.L., Eds.; Wageningen Academic Publishers: Waltham, MA, USA, 2010; pp. 233-249.

33. Brink, M.; Todorov, S.D.; Martin, J.H.; Senekal, M.; Dicks, L.M. The effect of prebiotics on production of antimicrobial compounds, resistance to growth at low $\mathrm{pH}$ and in the presence of bile, and adhesion of probiotic cells to intestinal mucus. J. Appl. Microbiol. 2006, 100, 813-820. 
34. Rhoades, J.; Gibson, G.R.; Formentin, K.; Beer, M.; Rastall, R.A. Inhibition of the adhesion of enteropathogenic Escherichia coli strains to HT-29 Cells in Culture by Chito-oligosaccharides. Carbohydr. Polym. 2006, 64, 57-59.

35. Shoaf, K.; Mulvey, G.L.; Armstrong, G.D.; Hutkins, R.W. Prebiotic galactooligosaccharides reduce adherence of enteropathogenic Escherichia coli to tissue culture cells. Infect. Immun. 2006, 74, 6920-6928.

36. Isolauri, E.; Salminen, S.; Ouwehand, A.C. Probiotics. Best Pract. Res. Clin. Gastroenterol. 2004, 18, 299-313.

37. Van den Ende, W.; Peshev, D.; de Gara, L. Disease prevention by natural antioxidants and prebiotics acting as ROS scavengers in the gastrointestinal tract. Trends Food Sci. Technol. 2011, 22, 689-697.

38. Capozzi, V.; Arena, M.P.; Crisetti, E.; Spano, G.; Fiocco, D. The hsp 16 gene of the probiotic Lactobacillus acidophilus is differently regulated by salt, high temperature and acidic stresses as revealed by reverse transcription quantitative PCR (qRT-PCR) analysis. Int. J. Mol. Sci. 2011, 12, 5390-5405.

39. Velasco, S.; Årskold, E.; Paese, M.; Grage, A.; Irastorza, A.; Rådstrom, P.; van Niel, E.W.J. Environmental factors influencing growth of and exopolysaccharide formation by Pediococcus parvulus 2.6. Int. J. Food Microbiol. 2006, 111, 252-258.

40. Bron, P.A.; Sally, M.; Hoffer, I.; van Swam, I.; de Vos, W.M.; Kleerebezem, M. Selection and characterization of conditionally active promoters in Lactobacillus plantarum, using alanine racemase as a promoter probe. Appl. Environ. Microbiol. 2004, 70, 310-317.

41. Bove, P.; Gallone, A.; Russo, P.; Capozzi, V.; Albenzio, M.; Spano, G.; Fiocco, D. Probiotic features of Lactobacillus plantarum mutant strains. Appl. Microbiol. Biotechnol. 2012, doi:10.1007/s00253-012-4031-2.

(C) 2012 by the authors; licensee MDPI, Basel, Switzerland. This article is an open access article distributed under the terms and conditions of the Creative Commons Attribution license (http://creativecommons.org/licenses/by/3.0/). 